\title{
RESEARCH-IN-BRIEF
}

Wie beurteilen unterschiedliche Meinungslager die Medienberichterstattung zur „Flüchtlingskrise“?

Ergebnisse einer Untersuchung zu Hostile-Media-Wahrnehmungen

How do persons with different opinions assess the media coverage of the "refugee crisis"?

Results of a study on hostile media perceptions

Milena Merten \& Marco Dohle 
Milena Merten (M.A.), Kommunikations- und Medienwissenschaft, Heinrich-Heine-Universität Düsseldorf, Universitätsstraße 1, 40225 Düsseldorf, Germany; Contact: milena.merten(at) gmail.com

Marco Dohle (PD Dr.), Kommunikations- und Medienwissenschaft, Heinrich-Heine-Universität Düsseldorf, Universitätsstraße 1, 40225 Düsseldorf, Germany; Contact: marco.dohle(at) phil.uni-duesseldorf.de 


\title{
RESEARCH-IN-BRIEF
}

\section{Wie beurteilen unterschiedliche Meinungslager die Medienberichterstattung zur „Flüchtlingskrise“? Ergebnisse einer Untersuchung zu Hostile-Media-Wahrnehmungen}

\author{
How do persons with different opinions assess the media coverage \\ of the "refugee crisis"? \\ Results of a study on hostile media perceptions
}

\section{Milena Merten \& Marco Dohle}

Zusammenfassung: Anhänger entgegengesetzter Positionen zu einem konflikthaltigen Thema tendieren dazu, die Medienberichterstattung zu diesem Thema als jeweils zuungunsten ihrer eigenen Position verzerrt wahrzunehmen. Dies wird als Hostile-Media-Effekt oder Hostile-Media-Wahrnehmung bezeichnet. In einer Befragungsstudie im Kontext der sogenannten Flüchtlingskrise wurde geprüft, wie Befürworter und Gegner einer flüchtlingsfreundlichen Willkommenskultur die inhaltliche Tendenz der Berichterstattung über das Thema wahrgenommen haben. Die Ergebnisse der Untersuchung zeigen, dass Hostile-Media-Wahrnehmungen bei beiden Gruppen auftreten, in besonderem Maße bei den Gegnern der Willkommenskultur. Die Wahrnehmungen korrelieren mit einem geringeren Vertrauen in die Medien, einer schlechteren Bewertung der Qualität der themenspezifischen Berichterstattung und erhöhter Wut.

Schlagwörter: Hostile-Media-Effekt, Wahrnehmungen, Ausgewogenheit, journalistische Qualität, „Flüchtlingskrise“

\begin{abstract}
Partisans on opposing sides of a conflict or controversial issue tend to perceive media coverage on this issue as biased against their own viewpoints. This is called hostile media effect or hostile media perception. In a survey study in the context of the so-called refugee crisis it was examined how supporters and opponents of a refugee-friendly culture of welcome perceived the tendency of media coverage on this topic. The results of the study show that hostile media perceptions occur in both groups, especially among the opponents of a refugee-friendly culture of welcome. The perceptions correlate with lower trust in the media, a worse evaluation of topic-specific reporting quality, and increased anger.
\end{abstract}

Keywords: Hostile Media Effect, Perceptions, Impartiality, Journalistic Quality, "Refugee Crisis” 


\section{Ausgangspunkt}

Die sogenannte Flüchtlingskrise beherrschte in den vergangenen Jahren in Deutschland viele politische Debatten sowie die Medienberichterstattung. Auch die Berichterstattung selbst war Gegenstand vieler Diskussionen. Im Kern ging es um die Frage, ob die Medien im Sinne einer Willkommenskultur berichtet haben oder ob sie dieser eher ablehnend gegenüberstanden: Haben sie humanitäre Verpflichtungen sowie Chancen betont oder diese ignoriert? Haben sie mögliche Herausforderungen und Probleme verharmlost oder diese im Gegenteil dramatisiert? Oder haben sie insgesamt unparteiisch und ausgewogen berichtet - und so ein zentrales Kriterium journalistischer Qualität erfüllt (z. B. Voigt, 2016, S. 61)?

Diese Fragen lassen sich durch Inhaltsanalysen beantworten (z. B. Haller, 2017; Maurer, Haßler, Jost, \& Kruschinski, 2018; Maurer \& Ringdal, 2017). Offen ist jedoch, ob sich deren Resultate mit Einschätzungen der Mediennutzer ${ }^{1}$ decken. Befragungen deuten darauf hin, dass dies nicht immer der Fall ist (z. B. Schultz, Jackob, Ziegele, Quiring, \& Schemer, 2017). Dies gilt vor allem für Personen, die bei einem starken Involvement eine dezidierte Meinung zu einem Thema haben. Sie nehmen Berichterstattung über dieses Thema verzerrt war und meinen häufig, die ihnen „feindlich“ gesinnte Gegenposition werde bevorzugt. Dies wird Hostile-Media-Effekt bzw. Hostile-Media-Wahrnehmung genannt (Vallone, Ross, \& Lepper, 1985).

1 Wenn in diesem Text für die Bezeichnung von Personengruppen die grammatikalisch maskulinen Formen genutzt werden, sind damit immer Menschen aller Geschlechtsidentitäten inkludiert.
Mit Hilfe einer Befragung wurde daher geprüft, wie Befürworter und Gegner der Willkommenskultur die inhaltliche Tendenz der Berichterstattung über die sogenannte Flüchtlingskrise wahrgenommen haben - und somit, ob unter den Befragten Hostile-MediaWahrnehmungen vorherrschen. Dadurch konnte ermittelt werden, welche Gültigkeit die Annahmen eines kommunikationswissenschaftlichen Ansatzes auch im Kontext der Berichterstattung zum vorliegenden Thema haben. Aus einer gesellschaftlichen Perspektive sind die Ergebnisse relevant, da etwaige Wahrnehmungen einer feindlich gesinnten Berichterstattung mit stärkerer Skepsis gegenüber journalistischer Qualität und mit Vertrauensverlusten einhergehen können (z. B. D’Alessio, 2003; Kim, 2011). Solche negativen Urteile, gerade wenn sie mit Blick auf wichtige und kontroverse Themen gefällt werden, können sich auf Bewertungen der Leistungen des gesamten Mediensystems ausweiten und als Folge sogar das Vertrauen in die Demokratie beeinträchtigen (Tsfati \& Cohen, 2005, 2013). Erkenntnisse zur Wahrnehmung von Berichterstattung helfen nicht nur, solche mutmaßlichen Probleme zu identifizieren, sondern sie liefern möglicherweise auch Hinweise, wie etwa der Journalismus reagieren kann.

\section{Theoretische Grundlage und Annahmen}

Als Hostile-Media-Effekt bzw. HostileMedia-Wahrnehmung wird das Phänomen bezeichnet, dass Anhänger einer bestimmten Position die Medienberichterstattung als zuungunsten dieser Position verzerrt wahrnehmen (Vallone 
et al., 1985). Nicht die generelle Wahrnehmung von Qualität steht somit im Vordergrund, sondern die wahrgenommene Ausgewogenheit.

Hostile-Media-Wahrnehmungen wurde in vielen Untersuchungen zu unterschiedlichen Themen und Ereignissen nachgewiesen - sowohl in Studien, in denen spezifische (meist ausgewogene) journalistische Beiträge beurteilt wurden, als auch in Studien, in denen die Tendenz der Gesamtberichterstattung zu einem Thema eingeschätzt werden sollte (Hansen \& Kim, 2011). Häufig werden Hostile-Media-Wahrnehmungen im Kontext von Konflikten überprüft, in denen sich zwei Gruppen mit entgegengesetzter Position gegenüberstehen. Gemäß der Kernannahme des Ansatzes sollten Vertreter beider Gruppen die Berichterstattung als feindlich gegenüber der eigenen Meinung wahrnehmen. Alternativ ist es möglich, dass Anhänger einer Position die Berichterstattung jeweils im Vergleich zu den Anhängern der Gegenposition für feindlicher bzw. weniger freundlich gegenüber ihrer eigenen Meinung halten - dies wird als relativer Hostile-Media-Effekt bezeichnet (z.B. Gunther \& Chia, 2001).

Auftreten und Intensität von Hostile-Media-Wahrnehmungen sind an Voraussetzungen geknüpft. Die Wahrnehmungen treten vor allem auf, wenn Individuen die Berichterstattung $\mathrm{zu}$ Themen einschätzen, die sie stark interessieren, die sie ihrer Auffassung nach gut beurteilen können und zu denen sie eine eindeutige Meinung vertreten. Dabei gilt: je eindeutiger die Meinung, desto stärker sollte die Hostile-MediaWahrnehmung ausfallen (Perloff, 2015; für weitere Erklärungsmuster z.B. Gunther \& Liebhart, 2006). Zudem wurden Folgen solcher Wahrneh- mungen geprüft. Dabei erwies sich, dass Hostile-Media-Wahrnehmungen unter anderem positiv korrelieren mit geringerem Vertrauen in Journalismus oder erhöhter Wut über Berichterstattung (z. B. Hwang, Pan, \& Sun, 2008; Kim, 2011; Tsfati \& Cohen, 2005).

Auch mit Blick auf Migrations- und Asylpolitikdebatten wurden bereits Hostile-Media-Studien durchgeführt (Beyer \& Matthes, 2015; McKeever, Riffe, \& Carpentier, 2012). Eine dieser Studien befasste sich direkt mit der aktuellen Debatte in Deutschland (Arlt \& Wolling, 2016, 2018). Erwartungsgemäß zeigten diese Untersuchungen: Je stärker viele der Befragten gegen Migranten eingestellt waren, desto eher nahmen diese Befragten eine wohlwollende Berichterstattung über Migration wahr. Positive Einstellungen zu Migranten gingen dagegen bei vielen Befragten einher mit Eindrücken einer migrationsfeindlichen Berichterstattung.

In diesen Studien lag der Fokus auf der Gesamtbevölkerung. Arlt und Wolling (2018) konnten indes in ihrer für deutschsprachige Online-User repräsentativen Stichprobe per Clusteranalyse zwei Gruppen identifizieren, die vergleichsweise starke Aktivitäten sowie eine entweder sehr positive oder sehr negative Einstellung gegenüber Flüchtlingen aufwiesen. Im Vergleich zu den anderen Befragten schätzten sie die Berichterstattung auch als aus ihrer Sicht feindlicher ein. In der vorliegenden Studie wurden solche Gruppen explizit in den Blick genommen und somit ausschließlich hochinvolvierte und in ihrer themenspezifischen Einstellung entgegengesetzte Personen angesprochen: auf der einen Seite Befürworter und auf der anderen Seite Gegner der Willkommenskultur (für genauere Angaben siehe Abschnitt 3.1). Es handelt sich hier- 
bei unter anderem deshalb um relevante Gruppen, da sie sich häufig in die öffentliche Debatte um Migration eingebracht und diese Debatte gewissermaßen mitbestimmt haben. Aufgrund ihrer starken Auseinandersetzung mit dem Thema wären eine intensive themenspezifische Mediennutzung und eine daraus resultierende differenzierte Betrachtung der Berichterstattung denkbar. Im Sinne des Hostile-Media-Effekts ist allerdings zu erwarten, dass Vertreter beider Gruppen die inhaltliche Tendenz der Berichterstattung eher als entgegengesetzt zu ihrer Meinung wahrnehmen. Je eindeutiger die Einstellung, desto stärker sollte der Eindruck sein, dass in der Berichterstattung gegenteilige Positionen vorherrschen. Daher gilt als Hypothese:

H1: (a) Je positiver die Einstellung gegenüber Flüchtlingen bei Willkommenskultur-Befürwortern und (b) je negativer die Einstellung bei Willkommenskultur-Gegnern, desto feindlicher wird die Tendenz der Berichterstattung zum Thema Flüchtlinge wabrgenommen.

Die Studie wurde einige Monate nach der Kölner Silvesternacht 2015/2016 durchgeführt. Diese Silvesternacht kann als Schlüsselereignis eingeordnet werden, in dessen Folge laut Inhaltsanalysen die Berichterstattung über Migration und Migrationsfolgen von größerer Skepsis geprägt war (z. B. Arendt, Brosius, \& Hauck, 2017; Budde, Jandura, \& Dohle, 2018). Dies ermöglichte es zu erfassen, ob dieser Berichterstattungswandel auch von Rezipienten wahrgenommen wurde. Solche etwaigen Veränderungen von Wahrnehmungen wurden in HostileMedia-Studien bislang nicht berücksichtigt. Anzunehmen ist, dass sich der
Eindruck feindlicher Medien unter den Willkommenskultur-Befürwortern spürbar verstärkt hat - die Berichterstattung ist nach der Silvesternacht migrationskritischer geworden, dies könnten migrationsfreundlich eingestellte Personen registriert haben. Willkommenskultur-Kritiker sollten dagegen nach der Silvesternacht eine nicht mehr ganz so stark ausgeprägte, aber immer noch feindlich-verzerrte Berichterstattung empfinden. Daher gilt:

H2: (a) Die Berichterstattung nach der Silvesternacht wird von Willkommenskultur-Befürwortern im Vergleich zu vorher als deutlich feindlicher wabrgenommen, (b) während Willkommenskultur-Gegner eine nur etwas weniger feindliche Berichterstattung wabrnehmen.

\section{Methode}

\subsection{Datenerhebung}

Zur Prüfung der Annahmen wurde von Mai-Juli 2016 eine standardisierte Online-Befragung unter Anhängern der beiden entgegengesetzten Meinungslager in der Flüchtlingsdebatte durchgeführt. Es wurden somit ausschließlich sogenannte Partisan-Gruppen befragt. Als Willkommenskultur-Befürworter wurden Interessengruppen identifiziert, die positiv gegenüber Flüchtlingen eingestellt sind und sich für deren Rechte und Schutz einsetzen (z. B. Menschenrechtsgruppen, Flüchtlingsräte, Willkommensbündnisse). Als Gegner der Willkommenskultur wurden Gruppen kontaktiert, die unter anderem verschärfte Asylverfahren fordern (z. B. AfD-Landesverbände, Pegida-Gruppen). Die Gruppen wurden zum einen per E-Mail mit der Bitte um Weiterlei- 
tung an ihre Mitglieder kontaktiert (wobei vielfach Absagen kamen). Zum anderem wurden Hinweise auf die Befragung in den jeweiligen FacebookGruppen veröffentlicht. Dieses Verfahren ist prinzipiell problematisch, da es vor allem zu einer Teilnahme thematisch stark interessierter Personen führt. Da in der Untersuchung jedoch gerade hochinvolvierte Personen von Interesse waren, stellt dies zunächst keine Einschränkung dar. Allerdings können die Ergebnisse nicht als repräsentativ für die Grundgesamtheit aller Partisans in der Flüchtlingsdebatte angesehen werden, zumal diese Grundgesamtheit weder bekannt ist noch klar definiert werden kann.

Für die Analysen wurden 468 Personen mit einer eindeutigen Einstellung zum Thema berücksichtigt (siehe $\mathrm{Ab}$ schnitt 3.2). Die Zahl der Gegner der Willkommenskultur $(n=358)$ überwog die Befürworterzahl $(n=110)$. Ein möglicher Grund hierfür: Bei den Facebook-Auftritten der Gruppen mit negativerer Haltung gegenüber Flüchtlingen waren oft Hinweise zur Studie erlaubt. Bei den Gruppen mit positiverer Haltung war dies fast immer verboten, fremde Beiträge wurden oft schnell gelöscht. Denkbar ist, dass die Gruppen schlechte Erfahrungen mit feindlichen Kommentaren gemacht haben und daher mit Fremdinhalten sehr strikt umgingen.

\subsection{Zentrale Variablen}

\section{Einstellung gegenüber Flüchtlingen.}

Trotz der gezielten Ansprache von Willkommenskultur-Befürwortern und -Gegnern wurde die Einstellung zu Flüchtlingen gesondert mit sieben Items gemessen (u. a. in Anlehnung an
Arlt \& Wolling, 2016). ${ }^{2}$ Die Items wurden zum Teil umcodiert und anschließend zu einem Einstellungs-Index verrechnet $(\alpha=0.95 ; 1=$ sehr negative Einstellung bis $5=$ sehr positive Einstellung). Personen mit einen Indexwert $\leq 2,5$ wurden als Gegner der Willkommenskultur definiert $(M=1.39$; $S D=.37)$, Personen mit einem Indexwert $\geq 3,5$ als Befürworter $(M=4.27$; $S D=.38) .^{3}$

Hostile-Media-Wabrnehmung. Die Tendenz der Berichterstattung musste differenziert nach Medien, Berichterstattungsaspekten und Zeitraum (vor/ nach Silvesternacht) eingeschätzt werden (für spezifischere Angaben und Ergebnisse: siehe Abschnitt 4).

Kontrollvariablen. Zur genaueren Stichprobenidentifikation wurden weitere Variablen erfasst. Sie dienten zudem als Kontrollvariablen in den Regressionen zu Hypothese 1. Die

2 Die Items lauteten: „Hat Deutschland durch die Zuwanderung von Flüchtlingen mehr Nachteile oder mehr Vorteile?" $(1=$ deutlich mehr Nachteile bis $5=$ deutlich mehr Vortei$l e)$; „Ich glaube, dass mit der Zuwanderung von Flüchtlingen die Sicherheit in Deutschland bedroht wird“; „Ich bin der Meinung, dass die kulturellen und/oder religiösen Differenzen zwischen den Flüchtlingen und den Deutschen zu groß sind“; „Ich finde, ein wirtschaftlich starkes Land wie Deutschland kann die angemessene Unterbringung und Versorgung der vielen Flüchtlinge gut leisten“; "Ich glaube, dass durch die verstärkte Zuwanderung von Flüchtlingen die Konkurrenz auf dem Wohnungsmarkt größer wird “; „Ich glaube, dass Flüchtlinge langfristig auf dem deutschen Arbeitsmarkt gebraucht werden“; „Ich sehe es als Deutschlands humanitäre Pflicht an, Flüchtlinge aus Krisengebieten aufzunehmen und angemessen zu versorgen" (jeweils 1 = stimme überhaupt nicht $z u$ bis $5=$ stimme voll und ganz $z u$ ).

3 Neben diesen 468 Personen gab es 31 Personen, die einen mittleren Indexwert erzielten. Sie wurden von den Analysen ausgeschlossen, da sie sich nicht eindeutig einem der beiden Meinungslager zuordnen ließen. 
Befragten lebten meist in den alten Bundesländern (Befürworter: 80.9\%; Gegner: 84.9\%). Die Willkommenskultur-Befürworter waren im Vergleich zu den Gegnern eher weiblich $(64.2 \%$ vs. $30.1 \%)$, jünger $(M=39.54$ Jahre, $S D=$ 14.07 vs. $M=48.77$ Jahre, $S D=$ 12.82), etwas höher gebildet (z. B. $17.6 \%$ vs. $26.3 \%$ mit höchstens Haupt-/Realschulabschluss), linker ( $M=$ $2.92, S D=1.53$ vs. $M=7.81, S D=$ $1.82 ; 1=$ links bis $11=$ rechts $)$ und häufiger persönlich in das Thema involviert $(M=3.19, S D=1.15$ vs. $M=$ $2.09, S D=1.02 ; 0=$ kein Involvement bis 5 = hohes Involvement). Ähnlich in beiden Gruppen waren Themeninteresse (Befürworter: $M=4.78, S D=0.48$; Gegner: $M=4.66, S D=0.90$ ) und allgemeines politisches Interesse (Befürworter: $M=4.39, S D=0.83$; Gegner: $M=4.60, S D=0.73$; jeweils: $1=g e-$ ringes Interesse bis $5=$ hohes Interesse). Zudem wurden die interne (Befürworter: $M=4.35, S D=0.74$; Gegner: $M=4.50, S D=0.82$ ) und die externe Efficacy erfasst (Befürworter: $M=$ 2.41; $S D=1.01$, Gegner: $M=1.45$, $S D=0.83$; jeweils: 1 = geringe Efficacy bis 5 = bohe Efficacy). ${ }^{4}$

\section{Ergebnisse}

Im Einklang mit der Grundannahme des Hostile-Media-Effekts unterscheiden sich Willkommenskultur-Befürworter und -Gegner in ihrem Eindruck von der Berichterstattung sehr deut-

4 Kaum Unterschiede offenbarten sich bei den Selbstauskünften zur themenbezogenen Mediennutzung. Einzig Online-Nachrichtenseiten sowie Social-Media-Kanäle wurden von Willkommenskultur-Befürwortern spürbar häufiger genutzt - dies lässt sich aber vermutlich auch durch ihr geringeres Durchschnittsalter erklären. lich. Dies zeigt sich zunächst mit Blick auf verschiedene Medien: Befürworter bescheinigen eine negative Darstellung von Flüchtlingen (Fernsehen: $M=$ $2.24, S D=0.85$; Online-Nachrichtenseiten: $M=2.24, S D=0.82$; Tageszeitungen/Zeitschriften: $M=2.28, S D=$ 0.83; Radio: $M=2.68, S D=0.65)$. Die Gegner nehmen eine äußerst flüchtlingsfreundliche Berichterstattung wahr (Fernsehen: $M=4.88, S D=0.37$; Online-Nachrichtenseiten: $M=4.63$, $S D=0.60$; Tageszeitungen/Zeitschriften: $M=4.66, S D=0.57$; Radio: $M=$ 4.74, $S D=0.51$; jeweils: 1 = viel zu negativ dargestellt bis $5=$ viel zu positiv dargestellt). Die Items lassen sich zu einem Index verdichten (Befürworter: $M=2.32, S D=0.67, \alpha=0.80$; Gegner: $M=4.74, S D=0.42, \alpha=0.82$; Mittelwertunterschied: $t(134.36)=$ $35.61 ; p<.001$ ).

Ähnliche Resultate ergeben sich bei einer Einschätzung von sechs unterschiedlichen Berichterstattungsaspekten (siehe Tab. 1). Die Items wurden in Anlehnung an die Items zur Messung der Einstellung gegenüber Flüchtlingen formuliert (siehe Fußnote 2). ${ }^{5}$

Zur eigentlichen Prüfung von Hypothese 1 wurden hierarchische Regressionen gerechnet (siehe Tab. 2). Als abhängige Variable diente der Index aus den vier Variablen zur Messung der medienspezifischen Wahrnehmungen die Werte wurden so umcodiert, dass

5 Für alle Items wurden einheitliche Skalenausprägungen verwendet. Die Ausprägungen „verharmlost" und „dramatisiert" sind bei den Items zum Arbeitsmarkt und zur humanitären Verpflichtung nicht ideal. Dennoch werden die Resultate präsentiert, weil sie zeigen, dass die Tendenz der Berichterstattung nicht nur mit Blick auf problematische Szenarien (siehe die ersten vier Items) zwischen den Gruppen unterschiedlich wahrgenommen wird. 
Tabelle 1. Wahrgenommene Tendenz mit Blick auf unterschiedliche Aspekte der Berichterstattung

\begin{tabular}{|c|c|c|c|c|c|}
\hline & \multicolumn{2}{|c|}{$\begin{array}{l}\text { Befürworter } \\
\text { Willkommens- } \\
\text { kultur } \\
(n=109-110)\end{array}$} & \multicolumn{2}{|c|}{$\begin{array}{l}\text { Gegner } \\
\text { Willkommens- } \\
\text { kultur } \\
(n=357-358)\end{array}$} & \multirow[t]{2}{*}{$\begin{array}{l}\text { Mittelwert- } \\
\text { unterschiede }\end{array}$} \\
\hline & $\mathrm{M}$ & SD & $\mathrm{M}$ & SD & \\
\hline $\begin{array}{l}\text { Die Bedrohung der Sicherheit in Deutschland } \\
\text { durch die Zuwanderung von Flüchtlingen }\end{array}$ & 4.45 & .75 & 1.17 & .49 & $\begin{array}{c}t(138.52)= \\
43.21 \\
p<.001\end{array}$ \\
\hline $\begin{array}{l}\text { Die kulturellen und/oder religiösen Differen- } \\
\text { zen zwischen Flüchtlingen und Deutschen }\end{array}$ & 4.27 & .82 & 1.05 & .24 & $\begin{array}{c}t(114.62)= \\
40.52 \\
p<.001\end{array}$ \\
\hline $\begin{array}{l}\text { Die Kosten und der organisatorische } \\
\text { Aufwand der Unterbringung und Versorgung } \\
\text { von Flüchtlingen }\end{array}$ & 3.97 & .91 & 1.23 & .67 & $\begin{array}{c}t(146.19)= \\
29.19 \\
p<.001\end{array}$ \\
\hline $\begin{array}{l}\text { Die verstärkte Konkurrenz auf dem Woh- } \\
\text { nungsmarkt durch die Zuwanderung von } \\
\text { Flüchtlingen }\end{array}$ & 3.64 & .92 & 1.32 & .57 & $\begin{array}{c}t(134.67)= \\
24.98 \\
p<.001\end{array}$ \\
\hline $\begin{array}{l}\text { Dass Flüchtlinge langfristig auf dem } \\
\text { deutschen Arbeitsmarkt gebraucht werden }\end{array}$ & 2.41 & .94 & 4.35 & 1.31 & $\begin{array}{c}t(248.42)= \\
-17.09 \\
p<.001\end{array}$ \\
\hline $\begin{array}{l}\text { Deutschlands humanitäre Verpflichtung, } \\
\text { Flüchtlinge aufzunehmen }\end{array}$ & 2.02 & .97 & 4.53 & 1.02 & $\begin{array}{l}t(465)= \\
-22.84 \\
p<.001\end{array}$ \\
\hline
\end{tabular}

Anmerkung. Mittelwerte auf einer Skala von $1=$ wurde von den Medien verharmlost bis $5=$ wurde von den Medien dramatisiert.

Quelle. Eigene Erstellung.

für jede Gruppe hohe Werte eine feindliche Verzerrung bedeuten und niedrige Werte eine Übereinstimmung mit der eigenen Einstellung. Zunächst wurden Kontrollvariablen berücksichtigt. Danach wurde die Indexvariable zur Messung der Einstellung gegenüber Flüchtlingen ergänzt. Einschlägige Werte verweisen darauf, dass keine Multikollinearität zwischen den Prädiktoren besteht (Toleranz: der geringste Wert liegt bei 0.4; VIF-Wert: der höchste Wert liegt bei 2.5). Die Analyse für die Willkommenskultur-Gegner zeigt: je negativer deren Einstellung gegenüber Flüchtlingen, desto feindlicher aus ihrer Sicht die Tendenz der Berichterstattung $(\beta=-.47 ; p<.001)$. Für die Willkommenskultur-Befürworter gilt: je positiver deren Einstellung gegenüber Flüchtlingen, desto negativer die wahrgenommene Tendenz der Berichterstattung gegenüber Flüchtlingen. Dieser Wert ist jedoch nicht signifikant $(\beta=$ $.17 ; p=.11$ ). Somit kann nur Hypothese $1 \mathrm{~b}$ klar bestätigt werden.

Die Befragten wurden zudem gebeten, die Tendenz der Berichterstattung differenziert für die Zeit vor und nach der Kölner Silvesternacht einzuschätzen. Während die WillkommenskulturBefürworter für die Zeit vor der Silvesternacht eine nur leicht negative Berichterstattung über Flüchtlinge unterstellen $(M=2.58, S D=.92)$, empfinden sie die Berichte danach als deutlich feindlicher $(M=1.42, S D=.67$; jeweils: 1 = viel zu negativ bis $5=$ viel 
Tabelle 2. Einfluss der Einstellung zum Thema Flüchtlinge auf die Wahrnehmung der Berichterstattung

\begin{tabular}{|c|c|c|}
\hline & \multicolumn{2}{|c|}{ Hostile-Media-Wahrnehmung } \\
\hline & $\begin{array}{l}\text { Befürworter } \\
\quad(n=105)\end{array}$ & $\begin{array}{l}\text { Gegner } \\
(n=322)\end{array}$ \\
\hline \multicolumn{3}{|l|}{ Kontrollvariablen: } \\
\hline Alter & 0.02 & $0.11^{*}$ \\
\hline Geschlecht $(1=$ weiblich $)$ & -0.06 & -0.05 \\
\hline $\begin{array}{l}\text { Bildungsgrad } \\
\text { (Referenz: kein Abitur): Abitur }\end{array}$ & -0.15 & -0.02 \\
\hline Hochschulabschluss & $-0.35 *$ & 0.02 \\
\hline Wohnort (1 = neue Bundesländer) & -0.04 & 0.08 \\
\hline Politisches Interesse & 0.25 & $0.13 *$ \\
\hline Ideologische Verortung (links ... rechts) & -0.03 & 0.01 \\
\hline Interne Efficacy & -0.11 & 0.04 \\
\hline Externe Efficacy & -0.01 & $-0.16 * *$ \\
\hline Interesse für das Thema Flüchtlinge & $-0.28 *$ & 0.01 \\
\hline Involvement & 0.15 & 0.00 \\
\hline$R^{2}$ & 0.14 & $0.17 * * *$ \\
\hline \multicolumn{3}{|l|}{ Unabhängige Variable: } \\
\hline Einstellungen zum Thema Flüchtlinge & 0.17 & $-0.47 * * *$ \\
\hline Veränderung $\mathrm{R}^{2}$ & 0.03 & $0.19 * * *$ \\
\hline$R^{2}$ gesamt & 0.16 & $0.36 * * *$ \\
\hline
\end{tabular}

Anmerkungen. Standardisierter Regressionskoeffizient $\beta$; Signifikanzlevel: ${ }^{*} p<0.05 ;{ }^{* *} p<0.01$; ${ }^{* * *} p<0.001$.

Quelle. Eigene Erstellung.

$z u$ positiv; vorher vs. nachher: $t(107)=$ $11.70 ; p<.001)$. Auch aus Sicht der Willkommenskultur-Gegner gab es nach der Silvesternacht $(M=4.47$, $S D=.74)$ Veränderungen im Vergleich zu vorher $(M=4.86, S D=.44$; vorher vs. nachher: $t(347)=9.35 ; p<.001)$. Allerdings wird die Berichterstattung als immer noch stark feindlich aufgefasst. Absolut gesehen erkennen die Willkommenskultur-Befürworter im Vergleich zu den Gegnern weitaus deutlichere Veränderungen $(t(146)=$ $.7 .17 ; p<.001)$. Die Ergebnisse sind somit im Einklang mit den Hypothesen $2 \mathrm{a}$ und $2 \mathrm{~b}$.

Schließlich wurden Verbindungen zwischen Hostile-Media-Wahrneh- mungen (Index der medienspezifischen Wahrnehmungen) und anderen Variablen geprüft: dem generellen Vertrauen in journalistische Angebote (Index aus vier Items; Befürworter: $M=3.09$, $S D=0.72, \alpha=0.80$; Gegner: $M=$ $1.50, S D=0.59, \alpha=0.76 ; 1=$ sehr geringes Vertrauen bis $5=$ sehr großes Vertrauen), der generellen Bewertung der Berichterstattung zur sogenannten Flüchtlingskrise (Index aus drei Items nach Kohring \& Matthes, 2004; Befürworter: $M=2.55, S D=0.88, \alpha=0.73$; Gegner: $M=1.30, S D=0.52, \alpha=$ 0.57; 1 = sehr schlechte Bewertung bis 5 = sehr gute Bewertung) sowie dem Ausmaß an Wut über die Berichterstattung (vor bzw. nach der Kölner Silves- 
ternacht; Befürworter: $M=3.11 / 4.15$, $S D=1.31 / 1.07$; Gegner: $M=$ 4.35/4.31, $S D=1.16 / 1.17 ; 1=$ sehr geringe Wut bis $5=$ sehr große Wut). Es zeigen sich Zusammenhänge zwischen Hostile-Media-Wahrnehmungen und einem geringeren Vertrauen (Befürworter: $r=-.37, p<.01$; Gegner: $r=-.52$, $p<.01)$, einer negativeren Bewertung der Qualität (Befürworter: $r=-.48, p<$ .01 ; Gegner: $r=-.45, p<.01)$ sowie erhöhter Wut (Befürworter: $r=.25, p<$ .05 sowie $r=.33, p<.01$; Gegner: $r=$ $.34, p<.01$ sowie $r=.30, p<.01$ ).

\section{Diskussion}

Die Untersuchung hat Limitationen: Die Zahl der Willkommenskultur-Befürworter ist trotz eines hohen Rekrutierungsaufwands gering. Allein schon aufgrund der Selbstselektion der Befragten handelt es sich nicht um repräsentative Stichproben derjenigen Bürger, die eine positive oder negative Einstellung gegenüber Flüchtlingen haben. Darüber hinaus legt die Rekrutierung über die Verteiler spezifischer Gruppen oder über deren FacebookAuftritte nahe, dass Personen teilgenommen haben, die mit Blick auf das Thema nicht nur hochinvolviert, sondern auch sehr aktiv sind. Diese Gruppen sind indes, unter anderem aufgrund ihrer vergleichsweise starken Aktivität, sehr relevant. Sie wurden in Hostile-Media-Studien zum Thema mit Ausnahme der gruppenspezifischen Analysen von Arlt und Wolling (2018) wenig bedacht.

Die Ausprägungen bei einigen Items zur Messung der Einstellungen gegenüber Flüchtlingen waren nicht ideal. Willkommenskultur-Gegner kritisierten zudem, dass nicht zwischen verschiedenen Flüchtlingsgruppen diffe- renziert wurde. Dies kann die Antworten zum Thema Flüchtlinge beeinflusst haben. Bei einer Differenzierung von Flüchtlingsgruppen hätte außerdem geprüft werden können, ob eine kritische Einstellung auch gegenüber politisch verfolgten Flüchtlingen vorliegt und die Wahrnehmung der Berichterstattung prägt - oder ob primär eine kritische Einstellung gegenüber anderen Migrantengruppen vorherrscht und für die Einschätzung der Berichterstattungstendenz wichtig ist.

Schließlich zeigen sich Zusammenhänge zwischen Hostile-Media-Wahrnehmungen und Variablen wie Vertrauen oder Wut, die aber aufgrund des Untersuchungsdesigns nicht kausal interpretiert werden können. Panelstudien wären eine Option, dieses Problem $\mathrm{zu}$ umgehen. Mit ihnen ließen sich auch etwaige Veränderungen der medienbezogenen Wahrnehmungen im Zeitverlauf prüfen.

Nichtsdestotrotz belegen die Resultate, in diesem Fall mit Blick auf die sogenannte Flüchtlingskrise, dass es der Journalismus als integrierende Kraft in Konflikten schwer hat - zumindest bei Rezipienten, die bei einer festen Einstellung stark in ein Thema involviert sind. Vielmehr werfen diese Rezipienten den Medien vor, parteiisch zu berichten, wobei sie stets ihre Meinung als unzureichend vertreten empfinden. Dies wurde bereits oft nachgewiesen, wobei sich die vorliegende Studie von vielen anderen Hostile-Media-Studien dahingehend unterscheidet, dass sie nicht experimentell angelegt war und mit einem natürlichen Stimulus außerhalb von Laborsituationen durchgeführt wurde.

Inhaltsanalysen kommen zu dem Ergebnis, dass deutsche Medien vor allem im Jahr 2015, also vor der Kölner 
Silvesternacht, eher positiv über Flüchtlinge berichtet und humanitäre sowie moralische Aspekte hervorgehoben haben, wobei Zuwanderung dennoch häufig als Gefahr und weniger als Chance betrachtet wurde (z. B. Maurer et al., 2018; Maurer \& Ringdal, 2017). Trotz dieser Tendenz ist die Deutlichkeit frappierend, mit der die Gegner der Willkommenskultur von einer aus ihrer Sicht feindlich gesinnten Berichterstattung ausgehen. Zudem offenbaren die sich diametral gegenüberstehenden Einschätzungen beider Gruppen, wie unterschiedlich Tendenzen medialer Berichterstattung wahrgenommen werden. Die Einschätzungen der Berichterstattungstendenz sind dabei unter den Willkommenskultur-Gegnern in starkem Maße von der Deutlichkeit ihrer Einstellung $\mathrm{zu}$ Flüchtlingen geprägt. Bei den Befürwortern spielt der Einstellungsgrad jedoch eine weitaus geringere Rolle. Andere Prädiktoren sind offenbar entscheidender. Die vorliegenden Ergebnisse weisen darauf hin, dass das Ausmaß an Interesse für Politik und Thema wichtig ist. Weitere mögliche Einflussfaktoren müssten in zukünftigen Studien getestet werden.

Hostile-Media-Wahrnehmungen können sich negativ auf das Bild vom Journalismus auswirken. Dies legen zum Beispiel die Zusammenhänge mit anderen Variablen nahe. In weiteren Studien wird zu überprüfen sein, ob sich eine Art Abwärtsspirale entwickelt, in der geringes Vertrauen, die Wahrnehmung feindlicher Berichterstattung sowie die schlechte Bewertung der Berichterstattungsqualität die wesentlichen Elemente ausmachen. Ebenso gilt es zu prüfen, ob HostileMedia-Wahrnehmungen auch konfliktverschärfende Folgen haben können.
Dies könnte der Fall sein, wenn Hostile-Media-Wahrnehmungen in einer zunehmenden Abschottung von als dissonant empfundenen Informationen resultieren - einhergehend mit einer stärkeren Zuwendung zu (oftmals tendenziösen) Alternativmedien, deren Rezeption möglicherweise Polarisierungsprozesse begünstigt (Del Vicario et al., 2016).

Die deutschen Medien haben ihre Berichterstattung zum Thema Flucht und Migration im Laufe der Zeit modifiziert. Sie berichten häufiger über problematische Aspekte, die direkt oder indirekt mit Migration in Zusammenhang gebracht werden - seltener hingegen über humanitäre Aspekte und die Schutzbedürftigkeit von Flüchtlingen (z. B. Arendt et al., 2017; Budde et al., 2018). Dies mag eine Folge von Ereignissen wie der Kölner Silvesternacht sein. Möglicherweise ist es aber auch eine Reaktion darauf, dass eine zu einseitige Berichterstattung vorgeworfen wurde. Sowohl Befürworter als auch Gegner der Willkommenskultur haben Veränderungen der Berichterstattung wahrgenommen. Offen ist, wie akkurat diese Wahrnehmungen sind, schließlich wurden sie mit nur wenigen Items und zudem mit einem recht großen zeitlichen Abstand zur Kölner Silvesternacht gemessen. Möglicherweise hat auch die Erwähnung der Silvesternacht aufgrund der Aufladung des Ereignisses in der politischen Diskussion dazu geführt, dass die Items suggestiv gewirkt haben und quasi nahelegten, von einer kritischeren Berichterstattung gegenüber Flüchtlingen nach der Silvesternacht auszugehen. Von den Willkommenskultur-Befürwortern wurden die Veränderungen der Berichterstattung jedenfalls stark kritisiert. Die Willkommenskultur-Gegner erkannten 
im Vergleich dazu leichtere Änderungen, sahen diese aber keineswegs als ausreichend an - wohl auch deshalb, weil Konfliktbeteiligte dezidiert eine Berichterstattung fordern, in der eindeutig ihre eigene Meinung vertreten wird (Arlt \& Wolling, 2018).

Es gibt viele Gründe, journalistische Berichterstattung - und somit auch die inhaltliche Tendenz dieser Berichterstattung - immer wieder zu überdenken und zu versuchen, sie zu verbessern. Die Ergebnisse dieser Studie deuten jedoch an, dass Veränderungen nicht allein das Ziel haben sollten, in Konflikten spezifischen Gruppen und Meinungslagern entgegenzukommen. Diese nehmen solche Veränderungen zwar wahr, honorieren sie aber nicht.

\section{Literatur}

Arendt, F., Brosius, H.-B., \& Hauck, P. (2017). Die Auswirkung des Schlüsselereignisses „Silvesternacht in Köln“ auf die Kriminalitätsberichterstattung [Investigating the consequences of the key event "New Year's Eve in Cologne" on crime reporting]. Publizistik, 62, 135152. https://doi.org/10.1007/s11616017-0322-z

Arlt, D., \& Wolling, J. (2016). The refugees: Threatening or beneficial? Exploring the effects of positive and negative attitudes and communication on hostile media perceptions. Global Media Journal, 6, 1-21.

Arlt, D., \& Wolling, J. (2018). Bias wanted! Examining people's information exposure, quality expectations and bias perceptions in the context of the refugees debate among different segments of the German population. Communications, 43, 75-99. https://doi.org/10.1515/ commun-2017-0045
Beyer, A., \& Matthes, J. (2015). Public perceptions of the media coverage of irregular immigration. Comparative insights from France, the United States, and Norway. American Behavioral Scientist, 59, 839-857. https://doi. org/10.1177/0002764215573253

Budde, N., Jandura, O., \& Dohle, M. (2018). Das Framing der Flüchtlingskrise in Parlament und Parlamentsmagazinen [The framing of the refugee crisis in parliament and parliamentary magazines]. Mitteilungen des Instituts für Deutsches und Internationales Parteienrecht und Parteienforschung (MIP), 24, 31-39.

D’Alessio, D. (2003). An experimental examination of readers' perceptions of media bias. Journalism \& Mass Communication Quarterly, 80, 282-294. https:// doi.org/10.1177/107769900308000204

Del Vicario, M., Bessi, A., Zollo, F., Petroni, F., Scala, A., Caldarelli, G., ... Quattrociocchi, W. (2016). The spreading of misinformation online. Proceedings of the National Academy of Sciences, 113, 554-559. https://doi.org/10.1073/ pnas. 1517441113

Gunther, A. C., \& Chia, S. C.-Y. (2001). Predicting pluralistic ignorance. The hostile media perception and its consequences. Journalism \& Mass Communication Quarterly, 78, 688-701. https:// doi.org/10.1177/107769900107800405

Gunther, A. C., \& Liebhart, J. L. (2006). Broad reach or biased source? Decomposing the hostile media effect. Journal of Communication, 56, 449-466. https://doi. org/10.1111/j.1460-2466.2006.00295.x

Haller, M. (2017). Die „Flüchtlingskrise“ in den Medien. Tagesaktueller Journalismus zwischen Meinung und Information [The „refugee crisis“ in the media. Journalism between opinion and information]. Frankfurt am Main: Otto Brenner Stiftung. 
Hansen, G. J., \& Kim, H. (2011). Is the media biased against me? A meta-analysis of the hostile media effect research. Communication Research Reports, 28, 169-179. https://doi.org/10.1080/0882 4096.2011.565280

Hwang, H., Pan, Z. D., \& Sun, Y. (2008). Influence of hostile media perception on willingness to engage in discursive activities: An examination of mediating role of media indignation. Media Psychology, 11, 76-97. https://doi. org/10.1080/15213260701813454

Kim, K. S. (2011). Public understanding of the politics of global warming in the news media: the hostile media approach. Public Understanding of Science, 20, 690-705. https://doi. org/10.1177/0963662510372313

Kohring, M., \& Matthes, J. (2004). Revision und Validierung einer Skala zur Erfassung von Vertrauen in Journalismus [Revision and validation of a trust in journalism scale]. Medien \& Kommunikationswissenschaft, 377-385.

Maurer, M., Haßler, J., Jost, P. B., \& Kruschinski, S. (2018). Auf den Spuren der Lügenpresse. Zur Richtigkeit und Ausgewogenheit der Medienberichterstattung in der „Flüchtlingskrise" [On the trail of the lying press. The correctness and balance of media coverage in the "refugee crisis"]. Vortrag gehalten auf der 63. Jahrestagung der DGPuK, Mannheim, 11. Mai 2018.

Maurer, P., \& Ringdal, S. (2017). The portrayal of populist and established parties in news and commentaries about immigration: An analysis of German and Norwegian newspapers in a pre-election period. Vortrag gehalten auf der ECREA Conference „Political Communication in Times of Crisis: New Challenges, Trends \& Possibilities “, Zürich, 23. November 2017.

McKeever, B. W., Riffe, D., \& Carpentier, F. D. (2012). Perceived hostile media bias, presumed media influence, and opinions about immigrants and immigration. Southern Communication Journal, 77, 420-437. https://doi.org/10.1080/1 041794X.2012.691602

Perloff, R. M. (2015). A three-decade retrospective on the hostile media effect. Mass Communication and Society, 18, 701-729. https://doi.org/10.1080/1520 5436.2015.1051234

Schultz, T., Jackob, N., Ziegele, M., Quiring, M., \& Schemer, C. (2017). Erosion des Vertrauens zwischen Medien und Publikum? Ergebnisse einer repräsentativen Bevölkerungsumfrage [Erosion of trust between the media and the audience? Results of a representative survey]. Media Perspektiven, (5), 246-259.

Tsfati, Y., \& Cohen, J. (2005). Democratic consequences of hostile media perceptions: The case of Gaza settlers. The Harvard International Journal of Press/ Politics, 10, 28-51. https://doi. org/10.1177/1081180X05280776

Tsfati, Y., \& Cohen, J. (2013). Perceptions of media and media effects: The third-person effect, trust in media and hostile media perceptions. In A. N. Valdivia \& E. Scharrer (Hrsg.), The international encyclopedia of media studies: Media effects/Media psychology (S. 5-19). Hoboken, NJ: Blackwell.

Vallone, R. P., Ross, L., \& Lepper, M. (1985). The hostile media phenomenon: Biased perception and perception of bias in coverage of the Beirut massacre. Journal of Personality and Social Psychology, 49, 577-585. https://doi. org/10.1037/0022-3514.49.3.577

Voigt, J. (2016). Nachrichtenqualität aus Sicht der Mediennutzer. Wie Rezipienten die Leistung des Journalismus beurteilen können [News quality from the perspective of media users. How recipients can evaluate the performance of journalism]. Wiesbaden: Springer VS. 